\title{
Detection of Human Papilloma Virus by means of Chromogen in situ hybridization (CISH)
}

\author{
Marjolein Schluck \\ FHML, Maastricht University \\ m.schluck@student.maastrichtuniversity.nl
}

\section{Abstracts}

Introduction. The human papillomavirus (HPV) can cause infections in humans, although most women will be infected with HPV at some time in their life only a few cases will progress into invasive disease. Of all the HPV types only the 'high-risk' viruses are associated with cervical carcinomas. In order to prevent cervical cancer, multiple countries have implemented a cervical cancer screening programme. The detection mechanism in these screening programmes are commonly based on the Papanicolaou test, colposcopic analysis and PCR. However the sensitivity of such tests have several limitations. Another detection tool is HPV in situ hybridization either with fluorescence (FISH) or with an enzyme reaction (CISH). The use of FISH ensures high sensitivity with low endogenous background. Furthermore, this method will result in high resolution and the possibility to quantify the signal intensity. However, the fluorescent signal fades after light exposure, and samples might have auto-fluorescence. Thus in a setting where routine analysis has to be carried out the CISH procedure is more convenient, as only a standard bright-field microscope is needed for the detection procedure. The aim of this thesis is to determine if there are enzymatic substrates that have stronger reaction products in comparison to the control substrate DAB. Secondly this thesis aims to determine which conjugate detection system results in the best localisation and least background. Finally, the main aim is to investigate the possibilities of developing such an CISH that the end staining is comparable or better than the detection with FISH. Materials \& Methods. Seven horseradish peroxidase substrates and six alkaline phosphatase substrates have been tested on CasKi cells with the centromere 1 and 1p36 probes as targets for the in situ hybridization reaction to determine the sensitivity and specificity of the substrates. The selected substrates were further tested with the HPV 16 probe as target on SiHa cells and on formalin-fixed and paraffin-embedded (FFPE) tissue sections to determine the best conjugate detection system. The DAB DAKO substrate and the FISH with FITC 
were used as controls. The selected substrate in combination with the selected conjugate detection system was then tested on a series of eighteen clinical samples. Results. The horseradish peroxidase substrates Vina Green and Seramun Grün resulted in superior precipitates in comparison to the DAB DAKO control. These substrates show very high sensitivity as the spots visible for 1 p3 6 were similar in size as the spots for $1 C$ of the control staining. The substrates Vulcan Fast Red and Ferangi Blue are the selected substrates for the alkaline phosphatase enzyme reaction. Both these substrates have good localised sensitive reaction products with, respectively, clear red and blue precipitate products. The Av-PO system in combination with the Vina Green substrate lead to the best results in comparison to the fluorescent control. All clinical samples tested with the Av-PO system tested positive for HPV infection and had the same sensitivity and specificity as the fluorescent samples. Discussion and Conclussion. The Av-PO system results in the same specificity and sensitivity on clinical samples as the FISH. However, the ease of localizing the infected area when only the integrated pattern is present is much better with FISH. In conclusion we can say that the Vina Green substrate in combination with the Av-PO systems targeting HPV in clinical samples gives similar results as FISH. For this reason we developed a CISH method which could be used in the routine setting for histopathological diagnosis.

\section{Keywords}

Human Papilloma Virus, Cervical Cancer, in situ hybridization.

\section{Introduction}

The human papillomavirus (HPV) can cause infections in humans, which can lead to a wide variety of cutaneous, mucocutaneous and mucosal proliferations(1). Although most women will be infected with HPV at some time in their life most of the infections will occur without clinical symptoms. Only a few cases will progress into invasive disease( 1 , 2). There are more than 100 types of the HPV known(3), of all these different types there are only 40 types that have the capacity to infect the genital tract $(3,4)$. Based on their clinical associations these 40 viruses can be divided into two groups: the 'low-risk' viruses, which generally contribute to the development of anogenital warts, these infections rarely develop into cancers, and the 'high-risk' viruses, these viruses are associated with lesions that are at high risk for malignant progression. Furthermore they are associated with almost all cervical carcinomas(5). Some of best known HPV types associated with anogenital cancers are HPV-16, 18, 31, 33, and 45(6). These 'high-risk' type viruses are prevalent throughout the human population, and are commonly transmitted via sexual 
contact(7). Of all cancers in women worldwide breast cancer and gynaecological cancers account for $52 \%$ of all prevalent cases(8). The gynaecological cancers, specifically cervical cancer, account for almost $12 \%$ of all cancers in women, thus representing the second most frequent cancer among women worldwide $(7,9)$. The majority of cervical cancer cases occurs in the developing world, about $83 \%$ of all cases(9). The phase after infection but before cervical cancer, the pre-malignant phase, can be divided into three different histological abnormalities; cervical intraepithelial neoplasia 1 (CIN1) which is a mild dysplasia, CIN2 which is classified as a moderate dysplasia, and $\mathrm{CIN}_{3}$ in this case severe dysplasia also known as carcinoma in situ is present(2).

In order to prevent the progression of cervical cancer, multiple countries have implemented a cervical cancer screening programme. The detection mechanism in these screening programmes are commonly based on the Papanicolaou test and colposcopic analysis. However the sensitivity of such tests have several limitations. Another detection tool is in situ hybridization either with fluorescent markers or with an enzyme reaction. The use of fluorescent molecules as markers ensures high sensitivity with low endogenous background. Furthermore this method will result in high resolution and the possibility to quantify the signal intensity. However the fluorescent signal fades after light exposure, and samples might have auto-fluorescence. Thus in a setting where routine analysis has to be carried out the enzymatic procedure is more convenient, as only a standard bright-field microscope is needed for the detection procedure. The aim of this bachelor thesis, therefore, is to determine which of the thirteen enzyme substrates, see materials and methods, leads to superior results in comparison the conventionally used 3,3'-Diaminobenzidine (DAB). In order to test this we decided to use the cell lines CasKi and SiHa with the targets centromere 1 (10000x1.7kb), 1p36 (6ookb), and HPV16 (8kb) as a model as their target seize is known. Furthermore, we aimed to determine which conjugate detection system will results in the best localisation and least background. Finally the main goal is to investigate the possibilities of developing such a standardized method that the end staining of the enzymatic in situ hybridization, on patient material, is comparable or better than the detection with fluorescence in situ hybridization.

\section{Materials and methods}

Seven horseradish peroxidase substrates and six alkaline phosphatase substrates have been tested on CasKi cells with the centromere 1 and ${ }_{1} \mathrm{P}_{3} 6$ probes as targets for the in situ hybridization reaction to determine the sensitivity and specificity of the substrates. 
The tested substrates for the HRP enzyme reaction are: AEC (PanPath, Netherlands), SermunGrün ${ }^{\circledR}$ chip (Seramun Diagnostica $\mathrm{GmbH}$, Germany), TrueBlue ${ }^{\mathrm{TM}}$ (Nutacon, Netherlands), DAB chromogen/Substrate kit (ScyTek, Utah) and the Romulin AEC Chromogen kit, Vina Green ${ }^{\mathrm{TM}}$ Chromogen kit, and Deep Space Black ${ }^{\mathrm{TM}}$ Chromogen kit (Biocare Medical, California). For the AP enzyme reaction the following substrates were tested: NBT/BCIP (PanPath, Netherlands), Alk-Phos Blue kit (ScyTek, Utah), New Fuchsin Kit (ScyTek, Utah) and Warp Red ${ }^{\mathrm{TM}}$ Chromogen kit, Ferangi Blue ${ }^{\mathrm{TM}}$ Chromogen kit 2, and Vulcan Fast Red Chromogen kit 2 (Biocare Medical, California). The substrate DAB DAKO (DAKO, DENMARK) was used as the control substrate which the sensitivity, background and intensity were compared to.

In order to determine the most sensitive conjugate detection system the selected substrates for HRP and AP were tested with a HPV 16 probe on the cell line SiHa and on Formalin-fixed and paraffin-embedded (FFPE) tissue sections to determine the best conjugate detection system. Again DAB (DAKO, Denmark) was used as a control as was FISH with FITC. The following conjugate options were tested:

- digoxigenin labelled probe - monoclonal mouse anti-digoxigenin(1:2000; Sigma) goat anti-mouse polymer of HRP or AP (Nichirei Biosciences, Japan)

- digoxigenin labelled probe - monoclonal mouse anti-digoxigenin(1:2000; Sigma) goat anti-mouse polymer of HRP(PolyTek)

- digoxigenin labelled probe - polyclonal rabbit anti-digoxigenin(1:5000; Sigma) - goat anti-rabbit polymer of HRP or AP (Nichirei Biosciences, Japan)

- biotin labelled probe - monoclonal mouse anti-biotin(1:100; DAKO, Denmark) - goat anti-mouse polymer of HRP or AP(Nichirei Biosciences, Japan)

- biotin labelled probe - avidine PO or AP(1:100; Vector) or FITC(1:500; Vector, California) - biotin labelled goat anti-avidine(1:100; Vector, California) - avidine PO or AP(1:100; Vector, California) or FITC(1:500; Vector, California)

- biotin labelled probe - avidine PO or AP(1:100; Vector, California)

- biotin labelled probe - anti-biotin antibody direct labelled with HRP or AP (PanPath, Netherlands).

The best substrate in combination with the best conjugate detection system was then tested on a series of eighteen clinical samples. Their fluorescent counterparts were used as control samples 


\section{Results}

\section{Substrate selection}

For the horseradish peroxidase enzyme reaction the substrates Vina Green and Seramun Grün resulted in stronger precipitates in comparison to the control (Figure 1A, B and C). These substrates showed very high sensitivity as the spots visible for 1p36 were similar in size as the spots for $1 \mathrm{C}$ of the control staining. However the Seramun Grün enzyme reaction did not develop into bright green precipitate products as was expected, on the contrary it formed dark green almost black spots. The Vina Green substrate on the other hand developed into, the expected, bright green spots. The substrates Vulcan Fast Red and Ferangi Blue were the substrates that have been selected for the alkaline phosphatase enzyme reaction (Figure $1 \mathrm{D}$ and $\mathrm{E}$ ). Both these substrates resulted in good localised sensitive reaction products in combination with the tested conjugate detection system with, respectively, clear red and blue precipitate products.

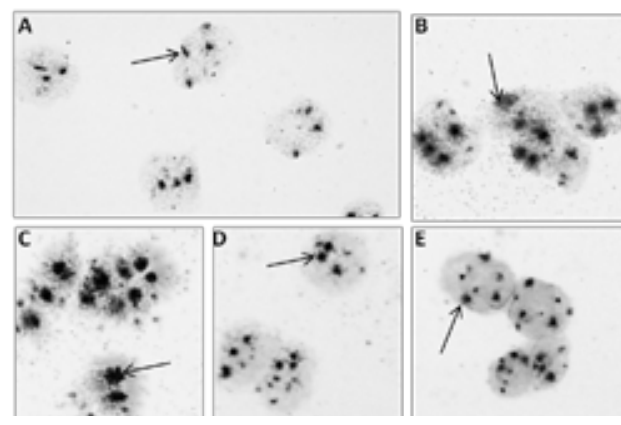

Figure 1. Centromere 1 visualized with the four best substrates in comparison to the DAB control. Pictures represent the CasKi cell line in which the centromere 1 has been stained using an ISH reaction. Pictures represent the DAB substrate $(A)$, the Vina Green substrate (B), Seramun Grün (C), the Ferangi Blue substrate (D), and the Vulcan Fast Red substrate (E) at a 40 times magnification and with a 1:4 heamtox counterstain. The arrows point towards a specific precipitate of centromere, the large spots.

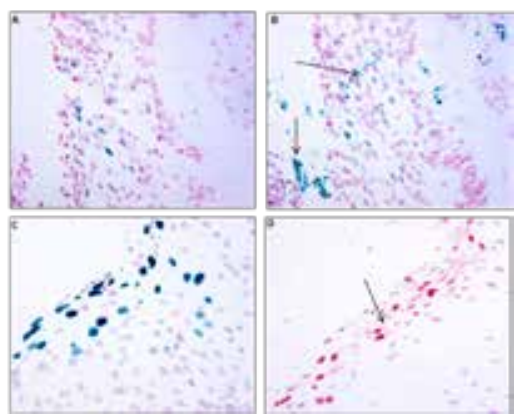

Figure 2. HPV16 visualisation with different conjugate systems. Representation of two different clinical samples of an HPV16 infection detected with different conjugate and substrate systems. Pictures represent the difference in background when using the three-layered Av-PO system (A) in comparison to the usage of the monoclonal anti-biotin antibody with the polymer as second layer(B), at a 40 times magnification. In both these samples the Nuclear Fast Red Counterstain (PanPath, Netherlands) has been used as opposed to a 1:4 heamatox counterstain (C and $D$ ). Figures $C$ and $D$ represent the same tissue section in which the HPV 16 has been visualized with the three 


\section{Conjugate detection system selection}

All the cell line samples using a polymer for the detection of the one copy of HPV 16 in SiHa cells showed high background compared to the fluorescent control. When the detection system avidine-PO - biotin labelled goat anti-avidine - avidine-PO (the Av-PO system) was used the results were even better than the fluorescent control. Different conjugate detection system have been tested with both the Vina Green and the Vulcan Fast Red substrates on clinical samples with a HPV16 infection.

The Vina Green substrate has been tested on FFPE tissue sections, with the Av-PO system, the monoclonal mouse anti-biotin system with the HRP polymer, and with a anti-biotin antibody directly labelled with HRP. The anti-biotin antibody directly labelled with HRP did not show any background, however there were also no clear signals visible. Both the Av-PO system and the HRP polymer did show signals. The polymer system, however, resulted in more background in the infected area than the Av-PO system (Figure $2 \mathrm{~A}$ and $\mathrm{B}$ ).

The Vulcan Fast Red substrate has been tested with the same detection systems but than for AP, in addition one layer of Av-AP has also been tested on tissue samples. The different detection systems were tested on FFPE tissue sections. For a comparison with Vina Green, this substrate has also been tested on the same tissue sample with the Av-PO system. Both the Av-AP system and the one layer of Av-AP did not show any signal nor background. The anti-biotin antibody directly labelled with AP produced very weak signals, only the nuclei that showed high positivity with the Vina Green substrate did show, to some extent, a red signal. The samples on which the AP polymer has been used had a visible bright red signal, however the intensity of the signal was less than with the Vina Green. Furthermore, the background in the stroma was higher (Figure $2 \mathrm{C}-\mathrm{D}$ ). For these reasons we used the Vina Green substrate on the series of eighteen clinical samples.
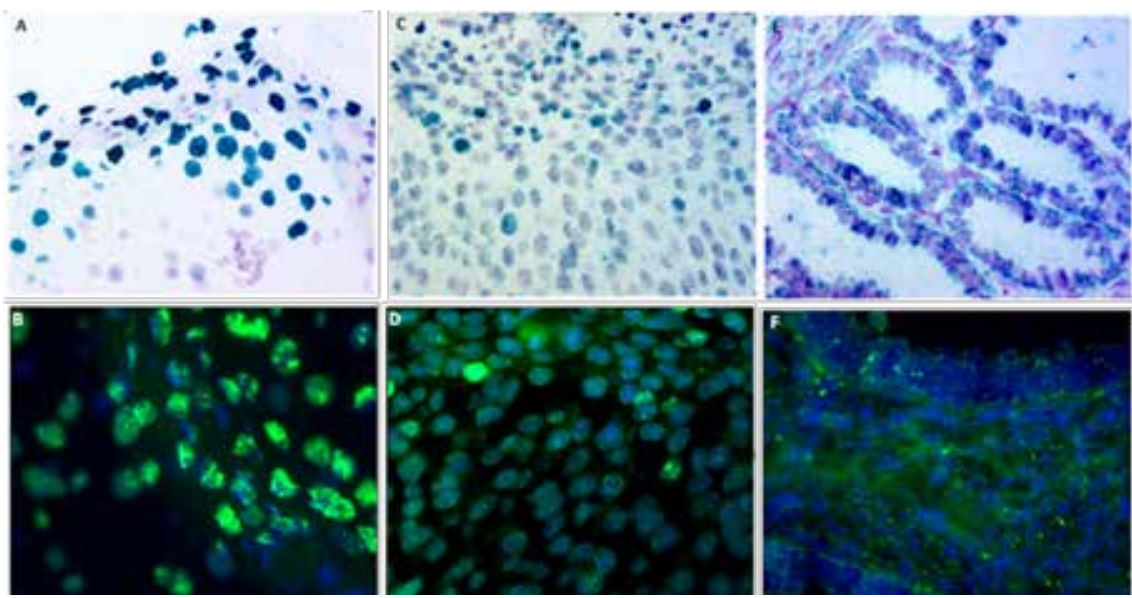
Figure 3. HPV16 visualization in clinical tissue section by using ISH and FISH. Images representing the ISH and FISH stainings of identical tissue sections (A-B, C-D, and E-F). The sections contain episomal (A-B), integrated (E-F), or both episomal and integraded (C-D) viral DNA. The episomal form of a HPV 31 infection visualised with ISH (A), at a 20 times magnification, compared to the fluorescent staining (B), at 40 times magnification with oil. Both the episomal and the integrated form of a HPV16 infection visualised with ISH (C), at 40 times magnification, compared to the fluorescent staining (D), at a 40 times magnification with oil. The integrated form of a HPV 16 infection visualised with ISH (E), at 40 times magnification, compared to the fluorescent staining (F), at 40 times magnification with oil. All ISH samples were counterstained with 1:4 heamtox and the FISH samples were counterstained with DAPI.

\section{HPV detection, Brightfield microscopy vs. Fluorescence microscopy}

To determine if the HPR polymer causes high background in more than one case, the polymer was tested on five of the clinical samples. In all the other remaining samples the Av-PO system was used. All five the samples tested with the polymer resulted in high background (like figure $2 \mathrm{~B}$ ). Due to this background it was not possible to determine positivity in two of the five samples. The thirteen samples for which the Av-PO system was used all tested positive for a HPV infection (Figure 3A, C, and D). When the clinical samples were compared to FISH analysed samples (already available) the sensitivity was the same. The individual spots visible with FISH were also visible with the Vina Green substrate (Figure $3 \mathrm{~A}$ till F).

\section{Discussion/Conclusion}

One of the aims of this thesis was to determine if there are substrates available which could potentially be used for the detection of HPV infection with brightfield microscopy in clinical samples. The tested substrates were only considered a potential candidate if their results on cell lines would result in stronger stainings than the staining of the DAB substrate, used as baseline control in this thesis. The substrates Seramun Grün (HRP), Vina Green (HRP), Vulcan Fast Red (AP), and Ferangi Blue (AP) were able to give some signal on the SiHa cell line staining for the HPV16 copy while the DAB staining could not. Therefore, it can be concluded that these substrates result in superior reaction precipitates in comparison to the $D A B$ staining and can be considered to be more sensitive than the $D A B$ substrate.

These selected substrates were further used to answer the second aim of this theses: what conjugate detection system provides the best localised precipitate with the least background staining? By testing the different conjugate detection systems the four substrates were further evaluated in order to determine the most sensitive substrate and detection system combination with the least background. The tests done on cell 
lines show that the monoclonal mouse anti-biotin in combination with the HRP polymer and Vina Green, the three-layered Av-PO system in combination with Vina Green, and the polyclonal rabbit anti-digoxigenin in combination with the AP polymer and Vulcan Fast Red are combinations of detection system and substrate which result into strong reactions. Despite the fact that the contrast with the red precipitate of the Vulcan Fast Red substrate is better with the hematoxylin counterstain than the contrast of the Vina Green with the hematoxylin counterstain, the Vina Green is considered to be superior to the Vulcan Fast Red. It might be possible that the goat anti-mouse AP polymer would lead to better results, when this polymer would become available again this needs to be tested. Contrary to the sensitive reaction of the Av-PO system, the Av-AP system does not show any reactivity at all.

In order to answer the main aim of this thesis, is it possible to develop an CISH method which results in comparable or better stainings than FISH, the Vina Green substrate had been chosen for testing on a series of eighteen clinical samples. These samples have been compared to the FISH stainings of the same eighteen tissue sections. When comparing the FISH samples with the Av-PO CISH counterparts the same specificity can be observed. The nuclei that are completely stained with the precipitate product, due to the presence of episomal viral DNA, can be observed with the lowest magnification without oil with both the brightfield $(4 \mathrm{X})$ microscope and with the fluorescence $(20 \mathrm{X})$ microscope. In order to detect the single spots of the integrated viral DNA a 20 times or 40 times magnification is needed in brightfield microscopy. With the fluorescence microscope the small individual spots are only visible when oil is applied at a 40 times magnification. In the case that a sample contains episomal viral DNA it can thus be easily spot with both the fluorescence and brightfield microscopes. However when a sample only contains integrated DNA, small individual spots, than the initial identification of the infected area is done more easily when using FISH. This is due to the fact that the small individual signals 'light-up' against a black background when the samples are stained with fluorescence, while with brightfield microscopy you need to search for the small spots in a counterstained tissue section. Nonetheless, when the infected area is found using the fluorescence microscope and the same area is examined with the brightfield microscope the small individual spots are also visible with the $\mathrm{CISH}$ procedure.

In summary, we found that the brightfield microscopy in combination with the Av-PO system with the substrate Vina Green delivers the same specificity and sensitivity as is observed with fluorescence microscopy. We noticed that the resolution of the brightfield 
microscopy can visualize the small individual spots using a 20 to 40 times magnification without the usage of oil. While for the fluorescence microscope, in case of an integrated pattern with small spots, oil is needed for the visualisation. However the ease of finding the infected area in a sample in which the viral DNA is only present in the integrated form is better when using a fluorescence microscope.

Concluding we can thus say that the Vina Green substrate in combination with the Av-PO systems targeting HPV in clinical samples gives similar results as the fluorescence in situ hybridization. For this reason we developed a CISH method which could be used in the routine setting for histopathological diagnosis.

\section{Role of the student}

Marjolein Schluck performed all the ISH and FISH reaction herself, except for the FISH staining of the clinical sample series, this was performed by Monique Ummelen of the department of Molecular Cell biology. The substrate selection part was performed by both Marjolein Schluck and Melanie Herre (fellow biomedical science student) both contributed equally, therefore this will be included in part in both our thesis's.

\section{References}

1. Zur Hausen H. Papillomavirus infections - a major cause of human cancers. Biochimica et Biophysica Acta (BBA)-Reviews on Cancer. 1996;1288(2):F55-F78.

2. Woodman CB, Collins SI, Young LS. The natural history of cervical HPV infection: unresolved issues. Nature Reviews Cancer. 2007;7(1):11-22.

3. De Villiers E-M, Fauquet C, Broker TR, Bernard H-U, zur Hausen H. Classification of papillomaviruses. Virology. 2004;324(1):17-27.

4. Munoz N, Castellsagué X, de González AB, Gissmann L. HPV in the etiology of human cancer. Vaccine. 2006;24:S1-S1O.

5. Werness BA, Levine AJ, Howley PM. Association of human papillomavirus types 16 and 18 E6 proteins with p53. Science. 1990;248(4951):76-9.

6. Jackson S, Storey A. E6 proteins from diverse cutaneous HPV types inhibit apoptosis in response to UV damage. Oncogene. 2000;19(4):592-8.

7. Zur Hausen H. Papillomaviruses and cancer: from basic studies to clinical application. Nature Reviews Cancer. 2002;2(5):342-50.

8. Pisani P, Bray F, Parkin DM. Estimates of the world-wide prevalence of cancer for 25 sites in the adult population. International journal of cancer. 2002;97(1):72-81.

9. Parkin DM, Bray F. The burden of HPV-related cancers. Vaccine. 2006;24:S11-S25. 